\title{
Measurements of Higgs boson properties in leptonic final states at CMS
}

\author{
Olena Hlushchenko*t \\ RWTH Aachen University \\ E-mail: olena.hlushchenko@cern.ch
}

The latest results obtained with CMS data collected at a centre-of-mass energy of $13 \mathrm{TeV}$ targeting Higgs boson decays into leptonic final states at CMS are discussed. The first differential cross section measurement of $H \rightarrow \tau \tau$, as well as the cross-sections measured for individual $\mathrm{ZH}$ and WH production mechanisms, is presented. The updated limits on anomalous vector boson couplings show no significant deviations from the standard model predictions.

European Physical Society Conference on High Energy Physics - EPS-HEP2019 -

10-17 July, 2019

Ghent, Belgium

* Speaker.

${ }^{\dagger}$ On behalf of the CMS Collaboration. 


\section{Introduction}

Following the observation of the Higgs boson [1-3] in 2012 a set of extensive analyses was carried out to study the properties of the newly discovered boson. An important role among those play the ones targeting Higgs boson decays into leptonic final states. After the first observation of the Higgs boson decays to tau leptons in 2016 [4] several studies were dedicated to closely look at the processes involving Higgs couplings to fermions. Recent results where the Higgs boson decays to a pair of tau leptons are discussed. All of them have used data collected in pp collisions at a centre-of-mass energy of $13 \mathrm{TeV}$ by the CMS detector [5] in 2016 while for the measurement of Higgs boson production and decay cross-sections additionally the 2017 dataset was used. These analyses target the main production mechanisms of the Higgs boson via gluon fusion $(\mathrm{ggH})$ and vector boson fusion $(\mathrm{VBF})$ while the production in association with a vector boson $(\mathrm{VH})$ is also considered in order to probe $\mathrm{WH}$ and $\mathrm{ZH}$ couplings directly. A closer study of Higgs boson properties as of today most precisely constrains anomalous HVV couplings, probing the $C P$ nature of the Higgs boson.

\section{Measurement of Higgs boson production and decay to the $\tau \tau$ final state}

The production cross section for the $H \rightarrow \tau \tau$ process is measured based on the data collected during the 2016 and 2017 data taking periods with a total integrated luminosity of $77.4 \mathrm{fb}^{-1}$ at $\sqrt{s}=13 \mathrm{TeV}$ [6]. The most sensitive final states of the di-tau system are used for the analysis: $e \mu, e \tau_{h}, \mu \tau_{h}$, and $\tau_{h} \tau_{h}$, where $\tau_{h}$ indicates a hadronic tau lepton decay. Up to $90 \%$ of the expected background contributions were estimated using data-driven methods. Those cover the processes where a jet is misidentified as a tau lepton ( $\mathrm{W}+\mathrm{jets}$, QCD, $t \bar{t}$, single $t$ and di-boson), and processes with genuine tau leptons in the final state $(Z \rightarrow \tau \tau, t \bar{t}$ production with two tau leptons in the final state, di-boson, and single $t$ ). The remaining backgrounds arising from events where an electron or muon is misidentified as a hadronic tau lepton decay $(Z \rightarrow l l, t \bar{t}$, single $t$ and di-boson processes) are estimated using simulation.

The fake-factor [7] method was used to constrain any processes where a jet is faking the hadronically

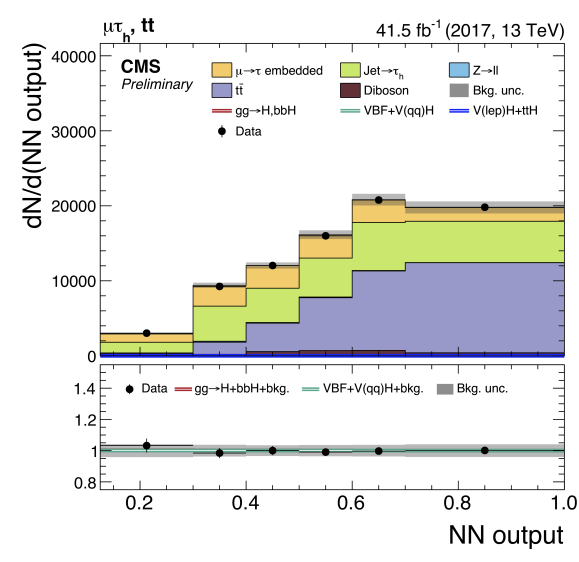

Figure 1: Distributions of the NN output used for the determination of the event categories in the $t \bar{t}$ event category, in the $\mu \tau_{h}$ final state as presented in [6]. All distributions and uncertainties are shown after the fit that has been applied to the data. decaying tau lepton. It relies on deriving the fake rates from the separate control regions populated by QCD, W+jets or $t \bar{t}$. The weighted average of the measured fake rates ("fake factors") is applied to events selected in the same way as those in the signal region but failing the tau lepton isolation requirement.

Contributions from backgrounds with genuine tau leptons in the final state, were constrained using $\tau$-embedded techniques described in [8]. The respective template (shown as " $\mu \rightarrow \tau$ " in 
Fig.1) subsumes as well $t \bar{t}$, di-boson, and single $t$ events containing genuine tau decays. In order to construct embedded samples, a pure set of $Z \rightarrow \mu \mu$ events is selected from data. Then any detector response associated with the two muons is removed on per-event basis and replaced with a simulated tau lepton. The tau lepton is generated such that its reconstructed 4-momentum would be the same as 4-momentum of the muon it is replacing.

The analysis categorisation is performed by using a multiclass neural network (NN) classification algorithm, providing a set of pure categories each targeting one respective signal or background process. Each event is assigned to a category with the highest NN score. For the statistical inference of the signal, a simultaneous binned maximum likelihood fit to of the NN score distributions in all event categories and all final states is performed, resulting in a fit based on 21 background and 8 signal categories ( $4 \mathrm{ggH}$ and $4 \mathrm{qqH}$ ). An example of such a category could be the $t \bar{t}$ category of the $\mu \tau_{h}$ channel for the 2017 dataset shown in Fig.1 where all the data points are located within the uncertainty bands showing a good understanding of the analysed data. The following cut-based categorisation based on the simplified template cross-section scheme (STXS), as defined by the LHC Higgs Cross Section Working Group [9], is necessary to provide the first differential cross-section measurement in $p_{T}^{\text {Higgs }}$ for $H \rightarrow \tau \tau$ decays.

For the inclusive Higgs boson production the product of the production cross-section and branching fraction into tau leptons is measured to be $\sigma^{i n c} B(\mathrm{H} \rightarrow \tau \tau)=2.56 \pm 0.48$ (stat) \pm 0.34 (syst) $\mathrm{pb}$. When resolved by production modes the products are found to be $\sigma(\mathrm{gg} \rightarrow$ $\mathrm{H}, \mathrm{bbH}) B(\mathrm{H} \rightarrow \tau \tau)=1.11 \pm 0.81$ (stat) \pm 0.78 (syst) $\mathrm{pb}$ and $\sigma(\mathrm{VBF}+\mathrm{V}(\mathrm{qq}) \mathrm{H}) B(\mathrm{H} \rightarrow \tau \tau)=0.34 \pm$ 0.08 (stat) \pm 0.09 (syst) pb. Here, "gg $\rightarrow \mathrm{H}, \mathrm{bbH}$ " stands for the gluon-fusion and production in association with a $b \bar{b}$ pair, while "VBF+V(qq)H" stands for electroweak qqH production, including both VBF and $p p \rightarrow V(\rightarrow q \bar{q}) H$ with hadronic $V \rightarrow q \bar{q}$ topologies. The results are presented in terms of constraints on individual signal strengths parameters and summarised in Fig.2.

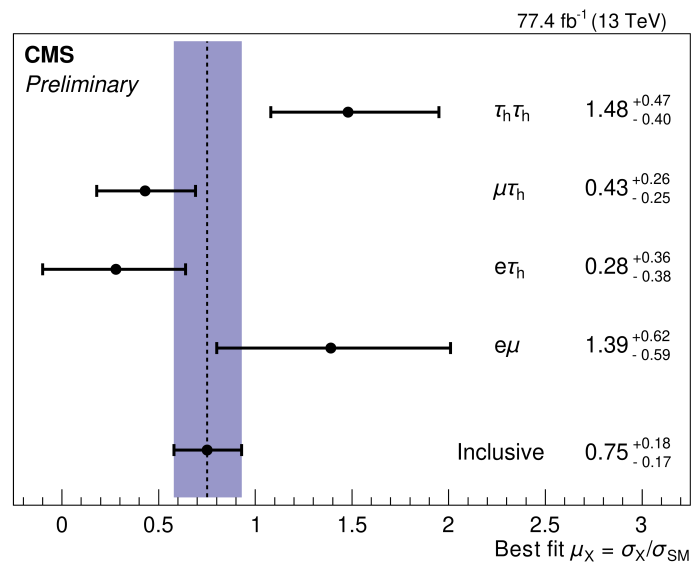

(a)

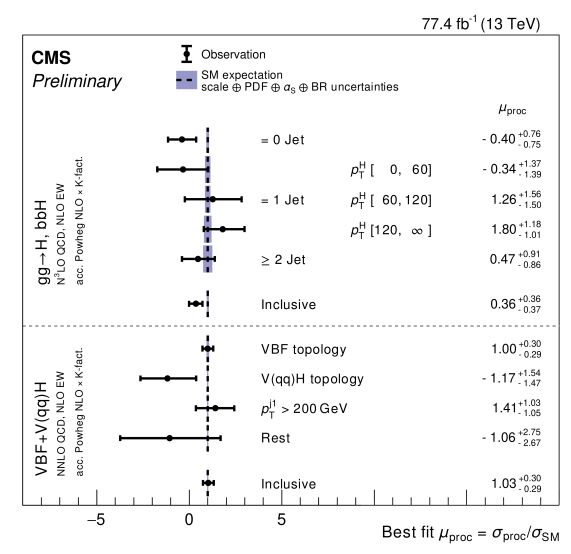

(b)

Figure 2: Constraints on the signal strength parameters obtained from a maximum likelihood fit inclusively and split into individual channels by final state (a), and split by production mechanism and differentially in $p_{T}^{H}$ for the $\mathrm{gg} \rightarrow \mathrm{H}$, bbH and $\mathrm{VBF}+\mathrm{V}$ (qq) $\mathrm{H}$ production processes (b) [6]. 


\section{Search for the associated production of the Higgs boson and a vector boson in proton-proton collisions at $\sqrt{s}=13 \mathrm{TeV}$ via Higgs boson decays to tau leptons}

A search for the standard model (SM) Higgs boson produced in association with a W (WH) or a $\mathrm{Z}(\mathrm{ZH})$ boson and decaying into a pair of tau leptons was performed following categorisation targeting respective productions based on three- $\left(l \mu \tau_{h}, l \tau_{h} \tau_{h}\right)$ and four-lepton $\left(l l e \tau_{h}, l l \mu \tau_{h}\right.$, $l l \tau_{h} \tau_{h}$ ) final states respectively [10]. The respective post-fit mass distributions are shown in Fig.3a and Fig.3b. The used data sample corresponds to an integrated luminosity of $35.9 \mathrm{fb}^{-1}$ and was collected in 2016 at a center-of-mass energy of $13 \mathrm{TeV}$.

For $\mathrm{ZH}$ production the categorization is done based on the scalar sum of $p_{T}$ of the visible decay products of the Higgs boson - $L_{T}^{\text {Higgs }}$. In the category with high $L_{T}^{\text {Higgs }}$ the large Higgs boson mass causes the decay products to have relatively high $p_{T}$ and leads to a higher signal purity as seen in Fig.3b. The optimized $L_{T}^{\text {Higgs }}$ threshold by signal sensitivity is equal to $50 \mathrm{GeV}$ in the lle $\mu$ final states, $60 \mathrm{GeV}$ in the $l l \mu \tau_{h}$ and $l l e \tau_{h}$ final states, and $75 \mathrm{GeV}$ in the $l l \tau_{h} \tau_{h}$ final state. The combined signal sensitivity for $\mathrm{VH}$ associated production is shown in Fig.3c.

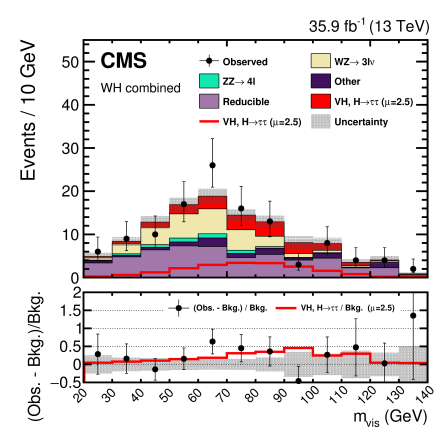

(a)

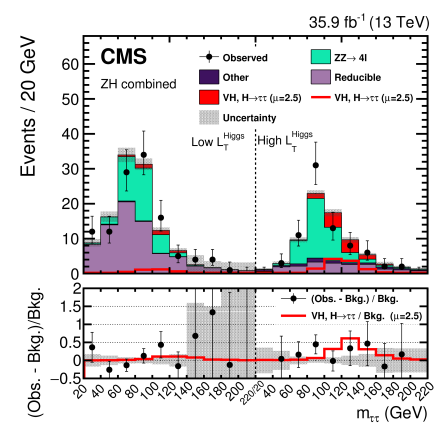

(b)

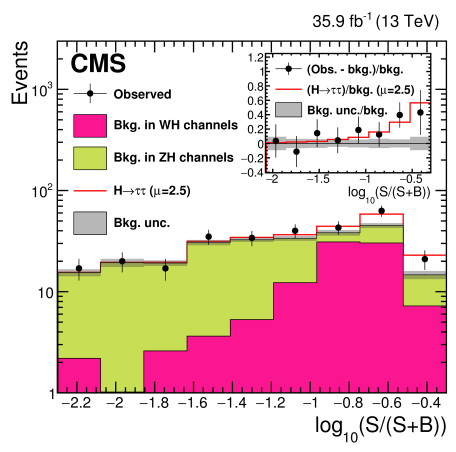

(c)

Figure 3: Post-fit mass distributions of combined final states for channels WH (a), ZH (b) and of the decimal logarithm of the ratio between the expected signal and the sum of the expected signal and background (c) as presented in [10]. The distribution in (b) is split into low and high $L_{T}^{H i g g s}$ regions. The uncertainties include both statistical and systematic components. The WH and $\mathrm{ZH}$ signal processes are summed together and shown as VH with its best fit signal strength value. The contribution from "Other" includes events from triboson, $t \bar{t}+W / Z, t \bar{t} H$ production, and all production modes leading to $H \rightarrow W W$ and $H \rightarrow Z Z$ decays.

The measured signal strength was found to be $\mu=3.39_{-1.54}^{+1.68}$ for $\mathrm{WH}, \mu=1.23_{-1.35}^{+1.62}$ for $\mathrm{ZH}$, and $\mu=2.5_{-1.3}^{+1.4}$ when added ( $\mathrm{VH}$ ). The post-fit mass distributions of final states for $\mathrm{WH}$ and $\mathrm{ZH}$, as well as the combined signal significance can be seen in Fig.3. The total VH signal template consists of $92 \%$ and $99 \%$ of WH (Fig.4a) and ZH (Fig.4b) in the respective categories.

A combination with earlier CMS measurements targeting ggH and VBF production modes for a Higgs boson with a mass of $125 \mathrm{GeV}$ was performed. The best observed (expected) fit signal strength is $\mu=1.24_{-0.27}^{+0.29}\left(1.00_{-0.23}^{+0.24}\right)$, and the observed (expected) significance is 5.5 (4.8) standard deviations as shown in Fig. 4a. The combination results in additional constraints on the vector bosons coupling of the Higgs boson. The measured couplings are consistent with the SM predictions within one standard deviation as seen in Fig. 4b providing increased confidence 


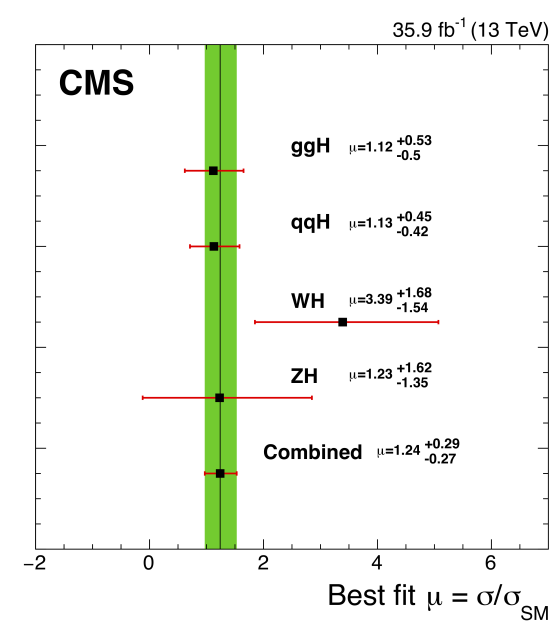

(a)

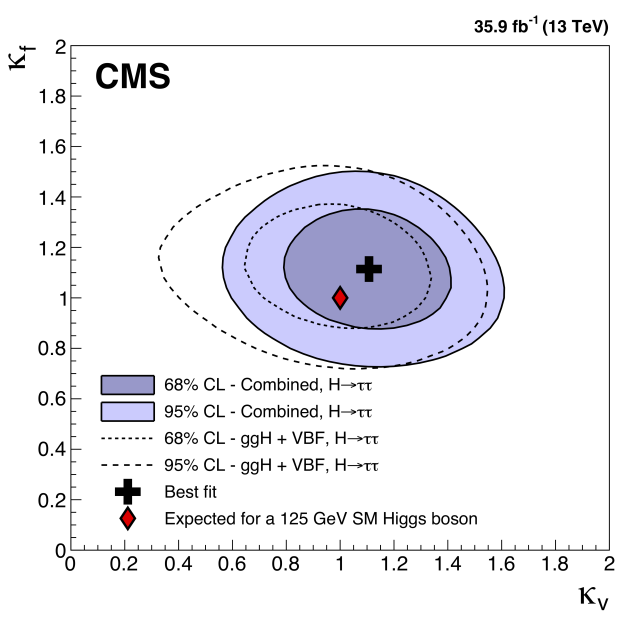

(b)

Figure 4: The observed best fit signal strengths per Higgs boson production process and their combination (a) and scans in $\kappa_{V}$ and $\kappa_{f}$ space of the negative log-likelihood difference, for $m_{H}=$ $125 \mathrm{GeV}$ (b) as described in [10]. The label "Combined" in (b) stands for a combination of the WH and $\mathrm{ZH}$ targeted analysis while results for the gluon-fusion and vector-boson-fusion analyses are labelled as "ggH + VBF".

that the Higgs boson couples to tau leptons through a Yukawa coupling as predicted in the SM. The combination allows for the extraction of the signal strengths for the four leading Higgs boson production processes using exclusively $H \rightarrow \tau \tau$ targeted final states, the results of which are largely consistent with the SM. This measurements of the Higgs boson production mechanisms using $H \rightarrow$ $\tau \tau$ decays are the best results to date for the $\mathrm{WH}$ and $\mathrm{ZH}$ associated production mechanisms.

\section{Constraints on anomalous HVV couplings from the production of Higgs bosons decaying to tau lepton pairs}

A study of anomalous HVV couplings was carried out on the data sample collected in the 2016 data taking period and corresponding to an integrated luminosity of $35.9 \mathrm{fb}^{-1}$, using Higgs boson candidates decaying into a pair of tau leptons and mainly produced in $\mathrm{VBF}, \mathrm{VH}$ and $\mathrm{ggH}$ processes [11].

Following the same formalism as the one used in a previous publication [12] and the theoretical approach described in [13], [14] anomalous interactions of a spin-0 Higgs boson with two spin-1 gauge bosons ( $W W, Z Z, Z \gamma$ and $g g$ ), are parametrized by a scattering amplitude that includes three tensor structures with expansion of coefficients up to $\left(q^{2} / \Lambda^{2}\right)$ :

$$
A(\mathrm{HVV}) \sim\left[a_{1}^{\mathrm{VV}}+\frac{\kappa_{1}^{\mathrm{VV}} q_{1}^{2}+\kappa_{2}^{\mathrm{VV}} q_{2}^{2}}{\left(\Lambda_{1}^{\mathrm{VV}}\right)^{2}}\right] m_{\mathrm{V} 1}^{2} \varepsilon_{\mathrm{V} 1}^{*} \varepsilon_{\mathrm{V} 2}^{*}+a_{2}^{\mathrm{VV}} f_{\mu \nu}^{*(1)} f^{*(2) \mu v}+a_{3}^{\mathrm{VV}} f_{\mu \nu}^{*(1)} f^{*(2) \mu \nu}
$$

where $q_{i}, \varepsilon_{V i}$, and $m_{V 1}$ are the four-momentum, polarization vector, and pole mass of the gauge boson (indexed by $\mathrm{i}=1,2$ ). The gauge boson's field strength tensor and dual field strength tensor are $f^{(i) \mu v}=\varepsilon_{V i}^{\mu} q_{i}^{v}-\varepsilon_{V i}^{v} q_{i}^{\mu}$ and $\tilde{f}_{\mu \nu}^{(i)}=\frac{1}{2} \varepsilon_{\mu v \rho \sigma} f^{(i) \rho \sigma}$. The coupling coefficients $a_{i}^{V V}$, which multiply 
the three tensor structures, and $\kappa_{i}^{\mathrm{V}} /\left(\Lambda_{1}^{\mathrm{VV}}\right)^{2}$, which multiply the next term in the $q^{2}$ expansion for the first tensor structure, are to be determined from data, where $\Lambda 1$ is the scale of beyond the SM (BSM) physics [11]. Effective fractional cross-sections $f_{a i}$ and phases $\phi_{a i}$ can then be expressed as:

$$
\begin{array}{rlrl}
f_{a 3} & =\frac{\left|a_{3}\right|^{2} \sigma_{3}}{\left|a_{1}\right|^{2} \sigma_{1}+\left|a_{2}\right|^{2} \sigma_{2}+\left|a_{3}\right|^{2} \sigma_{3}+\tilde{\sigma}_{\Lambda 1} /\left(\Lambda_{1}\right)^{4}+\ldots}, & \phi_{a 3}=\arg \left(\frac{a_{3}}{a_{1}}\right), \\
f_{a 2}=\frac{\left|a_{2}\right|^{2} \sigma_{2}}{\left|a_{1}\right|^{2} \sigma_{1}+\left|a_{2}\right|^{2} \sigma_{2}+\left|a_{3}\right|^{2} \sigma_{3}+\tilde{\sigma}_{\Lambda 1} /\left(\Lambda_{1}\right)^{4}+\ldots}, & \phi_{a 2}=\arg \left(\frac{a_{2}}{a_{1}}\right), \\
f_{\Lambda 1}=\frac{\tilde{\sigma}_{\Lambda 1} /\left(\Lambda_{1}\right)^{4}}{\left|a_{1}\right|^{2} \sigma_{1}+\left|a_{2}\right|^{2}{ }^{2}+\left|a_{3}\right|^{2} \sigma_{3}+\tilde{\sigma}_{\Lambda 1} /\left(\Lambda_{1}\right)^{4}+\ldots}, & \phi_{\Lambda 1}, \\
f_{\Lambda 1}^{Z \gamma}=\frac{\tilde{\sigma}_{\Lambda 1}^{Z \gamma} /\left(\Lambda_{1}^{Z \gamma}\right)^{4}}{\left|a_{1}\right|^{2} \sigma_{1}+\tilde{\sigma}_{\Lambda 1}^{Z \gamma} /\left(\Lambda_{1}^{Z \gamma}\right)^{4}+\ldots}, & \phi_{a i}^{Z \gamma},
\end{array}
$$

where $\sigma_{i}$ is the cross section for the process corresponding to $a_{i}=1$, and all other couplings are set to zero. The value of $f_{a 3}=1$ would correspond to the presence of pure pseudoscalar Higgs boson while any other non-zero value would imply a mixed $C P$ state.

The measurement of anomalous interactions relies on the optimal observables formed using the matrix element likelihood approach (MELA) [15]. Constraints are derived from the combination of the presented results with those from the $\mathrm{H} \rightarrow 4 l$ [16] decay channel are expressed as effective cross-section fractions and phases for $C P$-violating parameter $\left(f_{a 3} \cos \left(\phi_{a 3}\right)=(0.00 \pm 0.27) \times 10^{-3}\right.$ ) and $C P$-conserving parameters $\left(f_{a 2} \cos \left(\phi_{a 2}\right)=\left(0.08_{-0.21}^{+1.04}\right) \times 10^{-3}, f_{\Lambda 1} \cos \left(\phi_{\Lambda 1}\right)=\left(0.00_{-0.09}^{+0.53}\right) \times\right.$ $10^{-3}$, and $\left.f_{\Lambda 1}^{Z \gamma} \cos \left(\phi_{\Lambda 1}^{Z \gamma}\right)=\left(0.0_{-1.3}^{+1.1}\right) \times 10^{-3}\right)$. The $68 \%$ confidence level constraints are generally tighter than those from previous measurements.

The measurement of the $f_{a 3}$ parameter shows the results are consistent with expectations for the standard model H boson as seen in Fig.5.

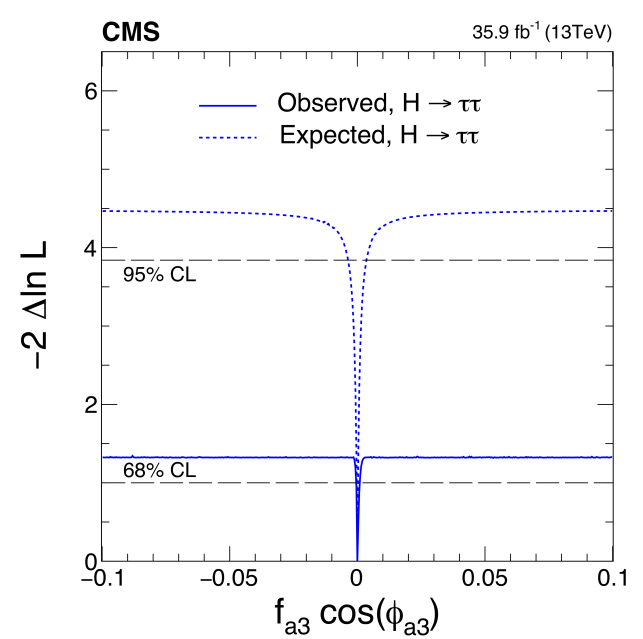

(a)

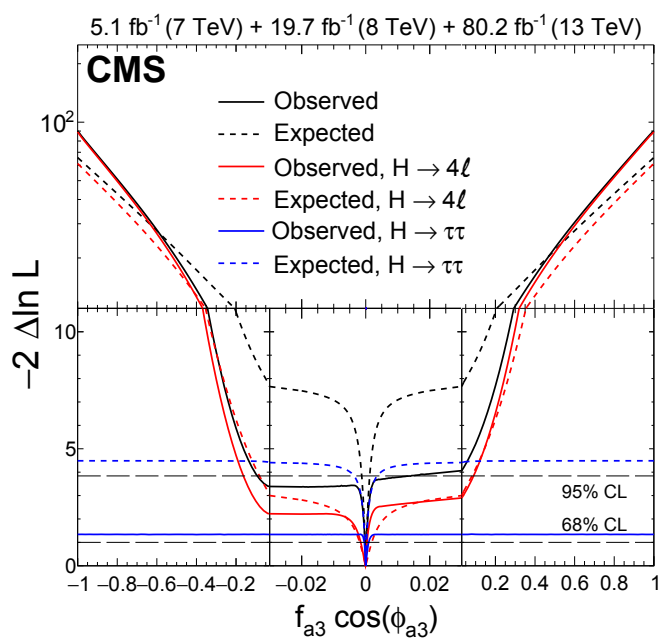

(b)

Figure 5: Observed and expected likelihood scans of $f_{a 3} \cos \left(\phi_{a 3}\right)$ standalone [11] (a) and in combination with $H \rightarrow 4 l$ [16] (b). 


\section{Conclusion}

The first differential cross-section measurements of $H \rightarrow \tau \tau$ were obtained, using the STXS formalism.

$H \rightarrow \tau \tau$ cross-sections are measured for individual $\mathrm{ZH}$ and $\mathrm{WH}$ production mechanisms as well as in combination with ggH and VBF.

The limits on anomalous couplings were updated. It is found that the analysed dataset does not allow for precise constraints on $C P$ properties.

\section{References}

[1] ATLAS Collaboration, Observation of a New Particle in the Search for the Standard Model Higgs Boson with the ATLAS Detector at the LHC, Phys. Lett. B 716 (2012) 1-29,

[doi:10.1016/j.physletb.2012.08.020], [arXiv:1207.7214]

[2] CMS Collaboration, Observation of a new boson at a mass of $125 \mathrm{GeV}$ with the CMS experiment at the LHC, Phys. Lett. B 716 (2012) 30-61, [doi:10 .1016/j.phys letb.2012.08.021], [arXiv:1207.7235]

[3] CMS Collaboration, Observation of a new boson with mass near $125 \mathrm{GeV}$ in pp collisions at ps $=7$ and 8 TeV, JHEP 06 (2013) 081, [doi : 10.1007 / JHEP 06 (2013) 081],, [arXiv: 1303.4571]

[4] CMS Collaboration, Observation of the Higgs boson decay to a pair of tau leptons with the CMS detector, CMS-HIG-16-043, [arXiv:1708.00373]

[5] CMS Collaboration, The CMS experiment at the CERN LHC, JINST 3 (2008) S08004, [doi:10.1088/1748-0221/3/08/s08004]

[6] CMS Collaboration, Measurement of Higgs boson production and decay to the $\tau \tau$ final state, CMS-PAS-HIG-18-032

[7] CMS Collaboration, Measurement of the $Z \gamma^{*} \rightarrow \tau \tau$ cross section in pp collisions at $\sqrt{s}=13$ TeV and validation of $\tau$ lepton analysis techniques, CMS-HIG-15-007, [arXiv:1801.03535]

[8] CMS Collaboration, An embedding technique to determine $\tau \tau$ backgrounds in proton-proton collision data, CMS-TAU-18-001, [arXiv:1903.01216]

[9] LHC Higgs Cross Section Working Group Collaboration, Handbook of LHC Higgs cross sections: 4. deciphering the nature of the Higgs sector, [doi:10.23731/CYRM-2017-002], [arXiv:1405.6569]

[10] CMS Collaboration, Search for the associated production of the Higgs boson and a vector boson in proton-proton collisions at $\sqrt{s}=13 \mathrm{TeV}$ via Higgs boson decays to $\tau$ leptons, CMS-HIG-18-007, [arXiv:1809.03590]

[11] CMS Collaboration, Constraints on anomalous HVV couplings from the production of Higgs bosons decaying to $\tau$ lepton pairs, CMS-HIG-17-034, [arXiv:1903.06973]

[12] CMS Collaboration, Measurements of the Higgs boson width and anomalous HVV couplings from on-shell and off-shell production in the four-lepton final state, [arXiv:1901.00174]

[13] T. Plehn, D. L. Rainwater, and D. Zeppenfeld, Determining the structure of Higgs couplings at the LHC, Phys. Lett. D 88 (2002) 051801, [doi: 10.1103 / PhysRevLett. 88 . 051801 ], [arXiv:01053254] 
[14] V. Hankele, G. Klamke, D. Zeppenfeld, and T. Figy, Anomalous Higgs boson couplings in vector boson fusion at the CERN LHC, Phys. Rev. D 74 (2006) 095001,

[doi:10.1103/PhysRevD.74.095001], [arXiv:hep-ph/0609075]

[15] Y. Gao et al., Spin determination of single-produced resonances at hadron colliders, Phys. Rev. D 81 (2010) 075022, [doi:10.1103/PhysRevD.81.075022], [arXiv:1001.3396]

[16] CMS Collaboration, Measurements of the Higgs boson width and anomalous HVV couplings from on-shell and off-shell production in the four-lepton final state, [arXiv:1901.00174] 